\title{
Knowledge, Attitude, and Practice on the Basic Life Support among Medical Interns of the Philippine General Hospital
}

\author{
Rogelio N. Velasco, Jr., MD, ${ }^{1}$ Mark David G. Francisco, MD² and Elmer Jasper B. Llanes, MD³ \\ ${ }^{1}$ Division of Medical Oncology, Department of Medicine, Philippine General Hospital, University of the Philippines Manila \\ ${ }^{2}$ Division of Endocrinology, Diabetes and Metabolism, Department of Medicine, Philippine General Hospital, University of the Philippines Manila \\ ${ }^{3}$ Division of Cardiology, Department of Medicine, Philippine General Hospital, University of the Philippines Manila
}

\begin{abstract}
Objective. This study aimed to assess the knowledge, attitude, and practice on the Basic Life Support (BLS) among the Philippine General Hospital medical interns, compare the scores between trained and untrained interns, and determine the proportion of respondents with updated BLS training.
\end{abstract}

Methods. This was a cross-sectional study using a thirty-five item questionnaire on the knowledge, attitude, and practice of medical interns at the Philippine General Hospital on the BLS.

Results. A total of 262 interns were included in the study. Although the mean knowledge scores of untrained and trained medical interns did not differ significantly ( 7.00 vs. $7.30, p=0.1637)$, more than $20 \%$ committed mistakes on questions on the following: jaw-thrust, rescue breaths, defibrillation, and CPR check. Generally, interns followed the BLS guidelines in their practice and had a positive attitude towards BLS. However, only $37 \%$ of trained participants had training within the past two years.

Conclusion. Most medical interns have a fair knowledge on BLS regardless of training status and adhere to them in practice. Since the training duration did not affect their knowledge scores, initial training in medical school and continuous exposure in the wards may have provided adequate BLS reinforcement. Recertification may not be needed during their formative years in medical school.

Key Words: basic life support, interns, KAP

\section{INTRODUCTION}

Basic Life Support is the core of resuscitation after sudden cardiac arrest. The major components of the Basic Life Support include recognition of cardiac arrest, activation of the EMS, initiation of high-quality CPR, and early defibrillation using an Automated External Defibrillator (AED). ${ }^{1}$

The newly-published guidelines on CPR in 2015 highlight a few significant changes, including the depth of chest compressions at 2-2.4 inches (compared to at least 2 inches previously) and the rate of chest compressions 100120 compressions per minute (compared to at least 100

Corresponding author: Rogelio N. Velasco, Jr., MD Division of Medical Oncology compressions per minute previously). The new guidelines Department of Medicine Philippine General Hospital University of the Philippines Manila Taft Avenue, Manila 1000, Philippines Email: rogervelascojr@gmail.com also underscore the importance of linking in-hospital cardiac arrest to out-of-hospital cardiac arrest. ${ }^{1}$

Locally, efforts have been initiated to increase the level of awareness on this pressing matter. Republic Act $10871^{2}$ was passed by the Philippine House of Representatives on 
July 17, 2016, targeting high school students in BLS training. Moreover, the Philippine Heart Association conducted a nationwide CPR training campaign last July 17, 2019. Despite efforts to increase the level of awareness among lay people, the goal of making the Philippines CPR-ready is still far from being reached.

Many studies have been undertaken to investigate the knowledge and skills of medical professionals on the BLS. A study by Afzalimoghaddam et al. in 2014 among 90 medical students in their sixth academic year evaluated their knowledge and skills before and after an objective structured clinical examination. Based on the results, the study concluded that routine BLS training in the medical curriculum could significantly increase medical students' knowledge of CPR administration. ${ }^{3}$ Moreover, knowledge of healthcare professionals on cardiopulmonary resuscitation has been correlated with spontaneous circulation return after in-hospital cardiac arrest.

To our knowledge, no study has been published regarding the knowledge, attitude, and practice of BLS among medical interns in the Philippines. This study aimed to assess the knowledge, attitude, and practice on the Basic Life Support among medical interns of the Philippine General Hospital, compare the scores between trained and untrained interns, and determine the proportion of respondents with updated BLS training.

\section{METHODS}

This cross-sectional study was conducted at the University of the Philippines - Philippine General Hospital, the largest tertiary referral center and government teaching hospital in the country with approximately 280 medical interns rotating, catering to around 250,000 patients a year. The study protocol with accompanying informed consent form was carried out upon approval from the University of the Philippines Manila Review Ethics Board (UPMREB). All interns rotating at the PGH were invited to participate in the study, regardless of certification status or medical school. Inclusion in this study was not a requirement for graduation, as indicated in the informed consent form. Since BLS certification has a validity of two years, interns were grouped as trained and untrained participants. Trained interns were defined as those who could obtain BLS training within the last two years, while the untrained interns were defined as participants without training within the previous two years.

\section{Written Questionnaire}

The questionnaire was developed using the 2015 Guidelines on adult BLS. The authors prepared the questionnaire in collaboration with Dr. Elmer Jasper Llanes, a member of the Philippine Heart Association National Expanded Council on Cardiopulmonary Resuscitation. The 35-item questionnaire was divided into three parts, namely: knowledge (14), attitudes (11), and practices (10). (Appendix) Questions included the rate and depth of chest compressions, proper sequence, the ratio of ventilation and chest compressions, proper administration of CPR, attitude, and medical interns' practices on the BLS. Other questions on their status of certification and frequency of performing BLS were also determined. The questionnaire was pretested among ten PGH medical interns who were chosen using a table of random numbers. Feedback regarding the content and adequacy of time appropriation were elicited and incorporated into the revised questionnaire.

Descriptive statistics were used to summarize the profile of the respondents. Frequency and proportion tables were utilized for nominal variables and mean and standard deviation for interval or ratio variables. Z-score for two means was used to compare the mean scores of trained and untrained samples. Fisher's exact test was utilized to compare the trained versus untrained interns' knowledge scores on individual item analysis while Mann Whitney $U$ test score was used to compare attitude and practice questions.

\section{RESULTS}

A total of 262 medical interns completed the questionnaire. The majority of the medical interns were female (55\%) and had BLS training (76\%). However, most interns' last training and certification were more than two years $(63 \%)$. (Table 1$)$

\section{Knowledge}

The mean scores of both trained and untrained participants did not differ significantly $(\mathrm{p}=0.1637)$. On individual item analysis, there was a statistically significant difference between those who had correct answers of trained and untrained respondents on question number 5 related to AED use $(p=0.013)$. For the rest of the knowledge

Table 1. Profile of the Participants

\begin{tabular}{lc}
\multicolumn{1}{c}{ Profile } & $\begin{array}{c}\text { Medical Intern }(\mathrm{n}) \\
\text { Mean, SD (\%) }\end{array}$ \\
\hline Age $(\mathrm{n}=244)$ & $25.77,1.66$ \\
\hline Gender $(\mathrm{n}=258)$ & \\
$\quad$ Female & $142(55)$ \\
Male & $116(45)$ \\
\hline BLS Training ( $=255)$ & \\
Yes & $194(76)$ \\
No & $61(24)$ \\
\hline Time since last BLS training $(\mathrm{n}=172)$ & \\
Within 6 months & $1(0.6)$ \\
Within 1 year & $13(7)$ \\
Within 2 years & $53(29)$ \\
More than 2 years & $115(63)$ \\
\hline Frequency of CPR practice $(\mathrm{n}=258)$ & \\
Daily & $4(1.55)$ \\
$>3 x$ per week & $46(18)$ \\
1-3x per week & $104(40)$ \\
<1x per week & $104(40)$ \\
\hline
\end{tabular}


Table 2. Distribution of Correct Knowledge Questions in Percentage According to Type of Respondents

\begin{tabular}{|c|c|c|c|c|c|}
\hline Knowledge Question No. & $\begin{array}{l}\text { Untrained } \\
\text { (n) }\end{array}$ & $\begin{array}{c}\text { Untrained } \\
\%\end{array}$ & $\begin{array}{l}\text { Trained } \\
\text { (n) }\end{array}$ & $\begin{array}{c}\text { Trained } \\
\%\end{array}$ & p-value \\
\hline Knowledge-1 (survey the scene) & 47 & 80 & 158 & 79 & 1 \\
\hline Knowledge-2 (jaw-thrust) & 43 & 73 & 152 & 76 & 0.606 \\
\hline Knowledge-3 (rescue breaths) & 41 & 69 & 144 & 72 & 0.62 \\
\hline Knowledge-4 (early chest compressions) & 52 & 88 & 178 & 89 & 0.812 \\
\hline Knowledge-5 (use of AED) & 34 & 58 & 149 & 75 & $0.013^{*}$ \\
\hline Knowledge-6 (rescue breaths) & 43 & 73 & 157 & 79 & 0.375 \\
\hline Knowledge-7 (ventricular fibrillation) & 27 & 46 & 102 & 51 & 0.553 \\
\hline Knowledge-8 (defibrillation) & 43 & 73 & 142 & 71 & 0.618 \\
\hline Knowledge-9 (chest compression rate) & 54 & 92 & 185 & 93 & 0.571 \\
\hline Knowledge-10 (CPR check after ventricular fibrillation) & 27 & 46 & 87 & 44 & 0.881 \\
\hline Mean Score and SD (total of 10 questions) & \multicolumn{2}{|c|}{$7.00(1.43)$} & \multicolumn{2}{|c|}{7.30 (1.50) } & 0.156 \\
\hline
\end{tabular}

${ }^{*} p$-value $<0.05$

Table 3. Knowledge on BLS Guidelines, RA 10871, and AED use among the Respondents

\begin{tabular}{lccc}
\multicolumn{1}{c}{ Knowledge Question No. } & $\begin{array}{c}\text { Choice 1: Yes, aware and read } \\
\mathbf{n}(\%)\end{array}$ & $\begin{array}{c}\text { Choice 2: Yes, aware but not Read } \\
\mathbf{n}(\%)\end{array}$ & $\begin{array}{c}\text { Choice 3: Not aware at all } \\
\mathbf{n}(\%)\end{array}$ \\
$\begin{array}{l}11 \text { - Awareness on New Guidelines } \\
\text { on BLS (2015) }\end{array}$ & $59(23)$ & $144(55)$ & $56(22)$ \\
\hline $\begin{array}{l}12 \text { - Awareness of RA 10871 BLS } \\
\text { Training in Schools Act }\end{array}$ & $57(22)$ & $116(45)$ & $87(33)$ \\
\hline Knowledge Question No. & $\begin{array}{c}\text { Choice 1: Yes } \\
\mathbf{n}(\%)\end{array}$ & $\begin{array}{c}\text { Choice 2: No } \\
\mathbf{n}(\%)\end{array}$ & $\begin{array}{c}\text { Choice 3: Not Sure } \\
\mathbf{n}(\%)\end{array}$ \\
\hline $\begin{array}{l}145(55) \\
\text { 14 - Ever seen an AED }\end{array}$ & $52(20)$ & $38(15)$ & $79(30)$ \\
\hline
\end{tabular}

questions, no difference was noted between the two groups. Notably, most participants committed a mistake on the rhythm check's proper timing $(54 \%$ and $56 \%$ for untrained and trained participants, respectively). Moreover, more than twenty percent committed mistakes in the following areas: jaw-thrust, rescue breaths, use of automated external defibrillators (AEDs), ventricular fibrillation, defibrillation, and CPR check. (Table 2)

Most of the respondents were aware of BLS's new guidelines (55\%) and RA 10871 (45\%) but had not yet read them. The majority have seen an AED (55\%) but have not used them (72\%). (Table 3)

\section{Attitude}

The majority of the interns disagreed that they should do mouth-to-mouth CPR when deemed necessary and that the BLS should be taught as early as elementary. (Table 4) The interns agreed on the following: needing more BLS training, ability to perform the BLS outside the hospital, including the BLS on the curriculum of non-medical courses. There was a significant difference between untrained and trained respondents on attitude question \#2 (need for BLS training) ( $\mathrm{p}=0.0132$ ) and attitude question \#11 (the belief that BLS should be trained as early as elementary) $(\mathrm{p}=0.0269)$. They strongly agreed on the following: providing $\mathrm{CPR}$ when the condition requires $\mathrm{CPR}$ is a life-saving procedure, medical students should have a review of BLS and training in ACLS, BLS should be part of the medical and non-medical courses, and teaching BLS as early as high school. As seen in this study, medical interns still believed they need more training, especially among the untrained ones. Furthermore, they also expressed a positive attitude toward learning ACLS.

\section{Practice}

The majority of the interns followed the current BLS recommendations on the following: frequency of rate of chest compressions, completing a full cycle without being replaced, allowing full chest recoil, maintaining a ninetydegree angle during $\mathrm{CPR}$, using the hip as a fulcrum during BLS, avoiding the bending of the elbow during $\mathrm{CPR}$, counting during $\mathrm{CPR}$, and reminding a member of the code team during actual CPR. (Table 5) There was no significant difference between groups except for practice question \#8 (avoiding excessive ventilation) ( $\mathrm{p}=0.037$ ).

\section{DISCUSSION}

The results of our study show that most medical interns have a fair knowledge of BLS. Also, the majority follow the guidelines in their practice and have a positive attitude towards BLS. A seminal review of the literature showed that this was the first to assess medical interns' knowledge, attitudes, and practices in the Philippines. 
Knowledge, Attitude and Practice on the Basic Life Support

Table 4. Distribution of Attitude Responses in Percentage According to Type of Respondents

\begin{tabular}{|c|c|c|c|c|c|c|c|}
\hline Attitude Question No. & Respondents & $\begin{array}{l}\text { Strongly } \\
\text { Disagree }\end{array}$ & Disagree & Neither & Agree & $\begin{array}{l}\text { Strongly } \\
\text { Agree }\end{array}$ & p-value \\
\hline \multirow[t]{2}{*}{ Attitude-1 (willingness to do BLS) } & Untrained & 0 & 0 & 5 & 34 & 58 & 0.201 \\
\hline & Trained & 1 & 0 & 0 & 32 & 67 & \\
\hline \multirow[t]{2}{*}{ Attitude-2 (feeling the need to have BLS training) } & Untrained & 0 & 0 & 10 & 36 & 53 & $0.0132^{*}$ \\
\hline & Trained & 1 & 5 & 11 & 47 & 36 & \\
\hline \multirow[t]{2}{*}{ Attitude-3 (willingness to do mouth-to-mouth) } & Untrained & 14 & 19 & 29 & 37 & 2 & 0.1609 \\
\hline & Trained & 16 & 34 & 19 & 24 & 8 & \\
\hline \multirow[t]{2}{*}{ Attitude-4 (ability to do BLS outside hospital) } & Untrained & 2 & 7 & 14 & 59 & 19 & 0.1437 \\
\hline & Trained & 1 & 4 & 8 & 67 & 21 & \\
\hline \multirow[t]{2}{*}{ Attitude-5 (believes that BLS is life-saving) } & Untrained & 0 & 0 & 0 & 19 & 81 & 0.7375 \\
\hline & Trained & 0 & 0 & 1 & 16 & 83 & \\
\hline \multirow{2}{*}{$\begin{array}{l}\text { Attitude- } 6 \text { (thinks that all medical interns should } \\
\text { have a review on BLS) }\end{array}$} & Untrained & 0 & 0 & 0 & 15 & 85 & 0.254 \\
\hline & Trained & 0 & 1 & 3 & 19 & 78 & \\
\hline \multirow{2}{*}{$\begin{array}{l}\text { Attitude-7 (believes that all interns should have } \\
\text { training on ACLS) }\end{array}$} & Untrained & 0 & 0 & 0 & 14 & 86 & 0.9217 \\
\hline & Trained & 0 & 0 & 0 & 13 & 87 & \\
\hline \multirow{2}{*}{$\begin{array}{l}\text { Attitude-8 (believes that BLS should be part of } \\
\text { the curriculum among medical courses) }\end{array}$} & Untrained & 0 & 0 & 2 & 8 & 90 & 0.2492 \\
\hline & Trained & 0 & 0 & 0 & 17 & 83 & \\
\hline \multirow{2}{*}{$\begin{array}{l}\text { Attitude-9 (believes that BLS should be part of } \\
\text { the curriculum among non-medical courses) }\end{array}$} & Untrained & 0 & 3 & 8 & 34 & 54 & 0.0577 \\
\hline & Trained & 2 & 2 & 11 & 47 & 39 & \\
\hline \multirow{2}{*}{$\begin{array}{l}\text { Attitude-10 (believes that BLS should be trained } \\
\text { as early as high school) }\end{array}$} & Untrained & 0 & 10 & 12 & 34 & 34 & 0.3665 \\
\hline & Trained & 0 & 10 & 18 & 37 & 29 & \\
\hline \multirow{2}{*}{$\begin{array}{l}\text { Attitude-11(believes that BLS should be trained } \\
\text { as early as elementary) }\end{array}$} & Untrained & 5 & 34 & 27 & 31 & 3 & $0.0269^{*}$ \\
\hline & Trained & 11 & 45 & 24 & 14 & 8 & \\
\hline
\end{tabular}

${ }^{*} p$-value $<0.05$

Table 5. Distribution of Practice Responses in Percentage According to Type of Respondents

\begin{tabular}{|c|c|c|c|c|c|}
\hline Practice Question No. & Respondents & Never & Sometimes & Always & p-value \\
\hline \multirow[t]{2}{*}{ Practice-1 (CPR rate) } & Untrained & 2 & 19 & 78 & 0.2421 \\
\hline & Trained & 2 & 27 & 71 & \\
\hline \multirow[t]{2}{*}{ Practice-2 (counting during CPR) } & Untrained & 15 & 53 & 31 & 0.3473 \\
\hline & Trained & 22 & 50 & 28 & \\
\hline \multirow[t]{2}{*}{ Practice-3 (instruction on rescuer) } & Untrained & 15 & 64 & 20 & 0.9821 \\
\hline & Trained & 20 & 56 & 25 & \\
\hline \multirow[t]{2}{*}{ Practice-4 (completing a cycle prior to replacing) } & Untrained & 7 & 25 & 68 & 0.6417 \\
\hline & Trained & 9 & 27 & 64 & \\
\hline \multirow[t]{2}{*}{ Practice-5 (pushing hard) } & Untrained & 0 & 27 & 73 & 0.9907 \\
\hline & Trained & 1 & 25 & 71 & \\
\hline \multirow[t]{2}{*}{ Practice-6 (allow recoil) } & Untrained & 0 & 20 & 78 & 0.3524 \\
\hline & Trained & 1 & 26 & 73 & \\
\hline \multirow[t]{2}{*}{ Practice-7 (arms at 90-degree angle) } & Untrained & 2 & 15 & 83 & 0.1379 \\
\hline & Trained & 2 & 25 & 73 & \\
\hline \multirow[t]{2}{*}{ Practice-8 (avoiding excessive ventilation) } & Untrained & 2 & 44 & 54 & $0.037^{*}$ \\
\hline & Trained & 4 & 57 & 39 & \\
\hline \multirow[t]{2}{*}{ Practice-9 (use of hips as a fulcrum) } & Untrained & 3 & 36 & 61 & 0.2925 \\
\hline & Trained & 0 & 33 & 67 & \\
\hline \multirow[t]{2}{*}{ Practice-10 (elbow bending) } & Untrained & 2 & 15 & 83 & 0.1365 \\
\hline & Trained & 1 & 26 & 73 & \\
\hline
\end{tabular}

${ }^{*} p$-value $<0.05$ 
The knowledge scores of the medical interns in this study were higher compared to other studies conducted. A study by Almesned et al. in 2014 among students and health providers in Qassim University concluded the poor knowledge of BLS among medicine, pharmacy, dentistry, and allied health science students and health providers. ${ }^{4}$ Moreover, Chandrasekaran et al. conducted a study in India among students, doctors and nurses of the medical, dental, homeopathy and nursing colleges which showed that almost $84.92 \%$ among the participants $(\mathrm{N}=1,054)$ attained less than $50 \%$ of the correct answers. ${ }^{5}$ However, a direct comparison in scores cannot be genuinely ascertained due to the different samples in these studies.

A study conducted by Yunus et al. in India among undergraduate-level medical and nursing students and junior doctors showed that the mean score for theoretical and practical knowledge among the participants significantly differed among the trained and untrained participants. ${ }^{6}$ This difference, however, was not seen in this study. Further studies with similar baseline characteristics may be done to ascertain the effect of training.

Although most interns have fair scores on the knowledge aspect, some aspects of the basic life support still need reinforcement, as shown in their mean knowledge scores (7.00 for untrained vs. 7.30 for trained). It is alarming that half of them have not read the new guidelines of basic life support. A study by Pillow et al. in 2014 among $1524^{\text {th }}$ year medical students in the US showed that students still feel unequipped in responding to emergency cardiac arrests and resuscitations and added that their medical school training could align their misconceptions and build their sense of readiness. ${ }^{7}$ A study conducted among medical students in Saudi Arabia showed that only 28\% of students attended BLS training in their final year. 51\% had poor knowledge (less than $60 \%$ ), while $44 \%$ had adequate knowledge $(60-80 \%) .{ }^{8}$ It must be noted that there was a trend towards a higher total mean score among the trained group compared to the untrained group $(\mathrm{p}=0.156)$.

Moreover, the proportion of interns who obtained corrected answers was also higher among the trained interns on most questions. The item on automated external defibrillators was significantly higher in the trained group $(\mathrm{p}=0.013)$. We surmise that the medical interns (both trained and untrained) may have been BLS-trained prior. This knowledge was indirectly reinforced through their rotations at the various wards by repeatedly attending to cardiac arrests. Furthermore, almost one-fourth of the interns were uncertified for the past two years.

The answers on the attitude and practice questions revealed both expected and unexpected findings. Untrained medical interns felt the need to have more BLS training, which is expected to be close to being licensed medical doctors. In general, most medical interns followed the BLS guidelines in their practice based on the questionnaire. Interestingly, untrained medical interns avoided excessive ventilation more than trained medical interns. However, these findings may be prone to bias since an actual practical exam was not undertaken. Hence, further evaluation needs to be conducted to assess BLS practice among medical interns fully.

\section{Limitations and future directions}

Although this study was conducted in 2017, no significant practice changes have been made on the BLS; thus, the questionnaire's content was still applicable. Since the study was questionnaire-based, medical interns' practice can be more fully assessed by a practical exam rather than through a questionnaire. The questionnaire utilized in this study may be validated, as well. Populations with similar baseline characteristics (baseline number of untrained or trained participants) may also be explored. A comparison of various health professionals (nurses, medical students in various years, residents) may also be investigated. Finally, qualitative studies like focus group discussions and interviews can be done to know more about the knowledge, attitude, and practices of health professionals regarding BLS.

\section{CONCLUSION}

Most medical interns have a fair knowledge on BLS regardless of training status and adhere to them in practice. Since the training duration did not affect their knowledge scores, initial training in medical school and continuous exposure in the wards may have provided adequate BLS reinforcement. Recertification may not be needed during their formative years in medical school.

\section{Statement of Authorship}

All authors participated in data collection and analysis, and approved the final version submitted.

\section{Author Disclosure}

All authors declare no conflicts of interest.

\section{Funding Source}

None.

\section{REFERENCES}

1. Neumar RW, Shuster M, Callaway CW, Gent LM, Atkins DL, Bhanji F, et al. Part 1: Executive Summary: 2015 American Heart Association Guidelines Update for Cardiopulmonary Resuscitation and Emergency Cardiovascular Care. Circulation. 2015 Nov;132 (18 suppl 2): S315-67. doi: 10.1161/CIR.0000000000000252.

2. Official Gazette of the Republic of the Philippines | The Official Gazette is the official journal of the Republic of the Philippines. Edited at the Office of the President of the Philippines Under Commonwealth Act No. 638 [Internet]. [cited 2017 Jan]. Available from: https://www.officialgazette.gov.ph/

3. Afzalimoghaddam M, Talebideloi M, Talebian MT, Farahmand S. Evaluation of the effectiveness of basic life support training on the knowledge and skills. Patient Saf Qual Improv. 2014; 2(2):73-6. doi: 10.22038/psj.2014.2518 
4. Almesned A, Almeman A, Alakhtar AM, AlAboudi AA, Alotaibi AZ, Al-Ghasham YA, et al. Basic life support knowledge of healthcare students and professionals in the Qassim University. Int J Health Sci. 2014 Apr; 8(2):141-50. doi: 10.12816/0006080.

5. Chandrasekaran S, Kumar S, Bhat S, Saravanakumar, Shabbir M, Chandrasekaran V. Awareness of basic life support among medical, dental, nursing students and doctors. Indian J Anaesth. 2010 Mar; 54(2):121-6. doi: 10.4103/0019-5049.63650.

6. Yunus Md, Mishra A, Karim H, Raphael V, Ahmed G, Myrthong C. Knowledge, attitude and practice of basic life support among junior doctors and students in a tertiary care medical institute. Int J Res Med Sci. 2015 Dec; 3(12):3644-50. doi: 10.18203/23206012.ijrms20151416

\section{APPENDIX}

\section{Questionnaire}

Date:

Age:

Sex:

Rotation:

BLS: Certified

o Yes

o No

(If yes) Date of last training?

\section{Knowledge}

1. A medical intern suddenly sees an elderly diabetic collapse in the middle of EDSA while crossing. What will be her first step in doing the Basic Life Support?
a. Check for responsiveness
b. Pulse check for not more than 10 seconds
c. Call for help
d. Survey the scene

2. An elderly woman sustained a cervical spine injury after a vehicular accident. As a volunteer, what maneuver should be done on our patient to open the airway?
a. Head tilt-chin lift maneuver
b. Jaw thrust without head-tilt
c. Airway should not be opened
d. A or B

3. A 25-year-old male sustained a gunshot wound at the chest after having an encounter with the police. Which of the following is false regarding the Basic Life Support based on the 2015 Guidelines?
a. Deliver rescue breaths every 6-8 seconds when the pulse is appreciated after five cycles of CPR
b. Deliver chest compressions at a depth of 2 to 2.4 inches
c. Deliver chest compressions at a rate of 100-140 compressions per minute
d. Pulse check is done on the carotid pulse

7. Pillow MT, Stader D, Nguyen M, Cao D, McArthur R, Hoxhaj S. Perceptions of basic, advanced, and pediatric life support training in a United States medical school. J Emerg Med. 2014 May;46(5):695-700. doi: 10.1016/j.jemermed.2013.08.055.

8. Raffaa H, Alshahrani A, Asiri AM, Mostafa O. Medical students' knowledge and practices related to basic life support. 2017.

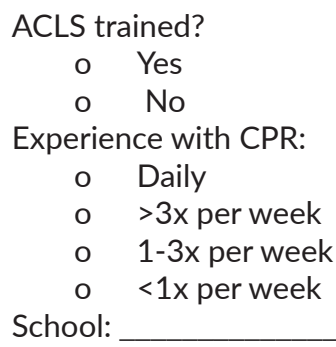

4. An elderly man complains of sudden chest pain and collapses on the floor. What is the best treatment for cardiac arrest until the arrival of ACLS care?
a. Early chest compressions
b. Administration of oxygen
c. Administration IV line
d. Administration of rescue breaths

5. Which of the following is true regarding the use of defibrillators:
a. For witnessed cardiac arrest when an AED is available, the defibrillator must be used as soon as possible.
b. When an AED is not immediately available, it is reasonable to start CPR while the device is being prepared, and then defibrillation is attempted as soon as possible.
c. Pulseless ventricular tachycardia is a shockable rhythm.

\section{d. All of the above}

6. Pulse check is done after five cycles of chest compression and rescue breaths. What is the ratio of chest compressions to ventilation?
a. 15 compressions: 2 rescue breaths
b. 30 compressions: 2 rescue breaths
c. 15 compressions: 1 rescue breath
d. 30 compressions: 1 rescue breath 
7. A medical intern having a vacation in Boracay suddenly sees an elderly diabetic complain of severe epigastric pain. He suddenly collapses on the floor. What is the most frequent initial rhythm in witnessed sudden cardiac arrest?
a. Asystole
b. Ventricular fibrillation
c. Ventricular tachycardia
d. Pulseless electrical activity

8. What is the treatment for ventricular fibrillation?
a. Cardioversion
b. Defibrillation
c. Adenosine
d. Verapamil

9. A code was called at the Medicine ward after the monitoring clerk note that the patient with decompensated heart failure on triple inotropes suddenly was found gasping. The code team should observe the following qualities of good CPR except:
a. Allow full recoil after each compression
b. Minimize pause between compressions
c. Compress to a depth of 2-2.4 inches
d. Compress at a rate less than 100 compressions per minute

10. A hypertensive uremic patient comes to the ER. ABG was severe high anion gap metabolic acidosis. Serum potassium noted was $8 \mathrm{mmol} / \mathrm{L}$. The monitoring intern calls for a code. CPR was initially done. On the cardiac monitor, ventricular fibrillation was seen. Shock was then delivered. What is the next step?

a. Check rhythm after delivering shock before doing CPR

b. Continue CPR and check rhythm after CPR cycle

c. Perform precordial thump

d. Deliver rescue breaths every 5-6 seconds

11. I am aware of the newly published guidelines in 2015 for Basic Life Support.

- Yes, aware and read

- Yes, aware and not read

o Not aware at all

12. I am aware of Republic Act No. 10871 Basic Life Support Training in Schools Act.

o Yes, aware and read

o Yes, aware but have not read

o Not aware at all
13. Have you ever seen an actual automated external defibrillator (AED)?

o Yes

o No

o Not sure

14. Have you ever used an automated external defibrillator (AED)?

o Yes

o $\mathrm{No}_{0}$

o Not sure

\section{Attitude}

1. If faced with a situation requiring it, I am willing to provide Basic Life Support.

o Strongly agree

o Agree

o Neither agree nor disagree

o Disagree

o Strongly disagree

2. I feel that I need more training in doing Basic Life Support.
o Strongly agree
o Agree
o Neither agree nor disagree
o Disagree
o Strongly disagree

3. I am willing to do mouth-to-mouth resuscitation if necessary.
- Strongly agree
o Agree
- Neither agree nor disagree
o Disagree
o Strongly disagree

4. I think I will be able to perform the Basic Life Support outside the hospital.

o Strongly agree

o Agree

o Neither agree nor disagree

o Disagree

o Strongly disagree

5. I think Basic Life Support is life-saving.

o Strongly agree

o Agree

- Neither agree nor disagree

o Disagree

o Strongly disagree 
6. I think all Medical Interns should have a review course on Basic Life Support before the Internship.

o Strongly agree

o Agree

- Neither agree nor disagree

o Disagree

o Strongly disagree

7. I think all Medical Interns should be trained in doing Advanced Cardiac Life Support in addition to BLS.

o Strongly agree

o Agree

- Neither agree nor disagree

o Disagree

o Strongly disagree

8. I think that Basic Life support should be part of the curriculum in medical courses.

o Strongly agree

o Agree

- Neither agree nor disagree

o Disagree

- Strongly disagree

9. I think that Basic Life support should be part of the curriculum in non-medical courses.

o Strongly agree

o Agree

o Neither agree nor disagree

o Disagree

o Strongly disagree

10. I think that Basic Life Support should be taught as early as high school.

o Strongly agree

o Agree

- Neither agree nor disagree

o Disagree

- Strongly disagree

11. I think that Basic Life Support should be taught as early as elementary.

o Strongly agree

o Agree

o Neither agree nor disagree

o Disagree

o Strongly disagree

\section{Practice}

1. When I do chest compression, I make sure it is at a rate of 100-120 per minute.
o Always
o Sometimes
o Never

2. I count during chest compressions.
o Always
o Sometimes
o Never

3. I instruct a rescuer to push hard and fast whenever I see them slowing down on chest compression.
o Always
o Sometimes
o Never

4. I complete a cycle of CPR and not asking to be replaced during CPR.
- Always
o Sometimes
o Never

5. I push hard (2 to 2.4 inches depth) during chest compressions.
o Always
o Sometimes
o Never

6. I allow full recoil after each chest compression.
- Always
o Sometimes
o Never

7. I maintain my arms at a 90-degree angle with respect to the patient's chest when I give chest compressions.
o Always
o Sometimes
o Never

8. I avoid excessive ventilation during CPR.
o Always
o Sometimes
o Never

9. I use my hips as the fulcrum when I do CPR.
o Always
o Sometimes
o Never

10. I do not allow my elbows to bend during CPR.
o Always
o Sometimes
o Never 